\title{
Multi-institutional comparison of treatment planning using stereotactic ablative body radiotherapy for hepatocellular carcinoma - benchmark for a prospective multi-institutional study
}

Takahisa Eriguchi ${ }^{1}$, Atsuya Takeda ${ }^{1}$, Yohei Oku ${ }^{1}$ Satoshi Ishikura ${ }^{2 *}$, Tomoki Kimura ${ }^{3}$, Shuichi Ozawa ${ }^{3}$, Takeo Nakashima ${ }^{3}$, Yukinori Matsuo ${ }^{4}$, Mitsuhiro Nakamura ${ }^{4}$, Yasuo Matsumoto ${ }^{5}$, Sadanori Yamazaki ${ }^{5}$, Naoko Sanuki and Yoshinori Ito ${ }^{6}$

\begin{abstract}
Introduction: Several single institution phase I and phase II trials of stereotactic ablative body radiotherapy (SABR) for liver tumors have reported promising results and high local control rates of over $90 \%$. However, there are wide variations in dose and fractionation due to different prescription policies and treatment methods across SABR series that have been published to date.

This study aims to assess and minimize inter-institutional variations in treatment planning using SABR for hepatocellular carcinoma (HCC) in preparation for a prospective multi-institutional study.

Methods: Four institutions (A-D) participated in this study. Each institution was provided with data from four cases, including planning and diagnostic CT images and clinical information, and asked to implement three plans (a practice plan and protocol plans 1 and 2). Practice plans were established based on the current treatment protocols at each institution. In protocol plan 1, each institution was instructed to prescribe 40 Gy in five fractions within 95\% of the planning target volume (PTV). After protocol plan 1 was evaluated, we made protocol plan 2, The additional regulation to protocol plan 1 was that $40 \mathrm{~Gy}$ in five fractions was prescribed to a $70 \%$ isodose line of the global maximum dose within the PTV. Planning methods and dose volume histograms (DVHs) including the median PTV D50 $\left(D_{m} 50\right)$ and the median normal liver volume that received $20 \mathrm{~Gy}$ or higher $\left(V_{m} 20\right)$ were compared.

Results: In the practice plan, $D_{m} 50$ was $48.4 \mathrm{~Gy}$ (range, 43.6-51.2 Gy). $V_{m} 20$ was 15.9\% (range, 12.2-18.9\%). In protocol plan 1, the $\mathrm{D}_{\mathrm{m}} 50$ at institution A was higher (51.2 Gy) than the other institutions (42.0-42.2 Gy) due to differences in dose specifications. In protocol plan 2, variations in DVHs were reduced. The $D_{m} 50$ was 51.9 Gy (range, 51.0-53.1 Gy), and the $\mathrm{V}_{\mathrm{m}} 20$ was $12.3 \%$ (range, 10.4-13.2\%). The homogeneity index was nearly equivalent at all institutions.
\end{abstract}

Conclusions: There were notable inter-institutional differences in practice planning using SABR to treat HCC. The range of PTV and normal liver DVH values was reduced when the dose was prescribed to an isodose line within the PTV. In multi-institutional studies, detailed dose specifications based on collaboration are necessary.

Keywords: Stereotactic body radiotherapy, Stereotactic ablative body radiotherapy, SBRT, SABR, Benchmark, Clinical study, Hepatocellular carcinoma

\footnotetext{
* Correspondence: s_ishiku@juntendo.ac.jp

${ }^{2}$ Department of Radiation Oncology, Juntendo University, Tokyo, Japan

Full list of author information is available at the end of the article
} 


\section{Introduction}

Hepatocellular carcinoma (HCC) primarily affects patients with chronic liver disease. Patients with chronic hepatitis or cirrhosis secondary to viral hepatitis B or C and alcoholism are at the highest risk of developing HCC. Clinical practice guidelines [1,2] recommend surgical resection, transplantation or percutaneous ablation to treat solitary $\mathrm{HCC}$ in patients with adequate liver function.

Stereotactic ablative body radiotherapy (SABR) is an emerging treatment modality that enables delivery of ablative doses to tumors with acceptable toxicity. Several single institution phase I and phase II trials of SABR for liver tumors have reported promising results and high local control rates of over 90\% [3-6]. Additional multiinstitutional prospective studies could establish this as an alternative treatment for patients who are ineligible for other local treatments for solitary HCC. However, there are wide variations in dose and fractionation due to different prescription policies and treatment methods across SABR series that have been published to date [3,4,7-9].

We assessed inter-institutional variations in SABR planning to treat $\mathrm{HCC}$ and run a benchmark in preparation for a multi-institutional prospective study.

\section{Methods}

\section{Study schemes}

Four institutions (A, B, C and D) participated in this study. Anonymized data from four benchmark cases with HCC were distributed to the participating institutions, including planning computed tomography (CT) images, pretreatment triphasic $\mathrm{CT}$ images and clinical information. Planning CT images from each case are shown in Figure 1. The tumors were in different locations with maximum tumor diameters of 22, 23, 25 and $40 \mathrm{~mm}$. Structure sets of the liver, gross tumor volume (GTV), internal target volume (ITV) and planning target volume (PTV) were also provided. The PTV of case 1, 2, 3, and 4 were 35.4, 50.5, 87.6 and $105.8 \mathrm{cc}$, respectively. Pretreatment triphasic CT images were acquired at a resting expiratory level with the patient in a vacuum pillow and under abdominal compression. Planning CT images were acquired in a long scan (6-8 seconds/slice) during free breathing. GTV was contoured on pretreatment triphasic $\mathrm{CT}$ and combined with planning CT. CTV was equated to GTV. ITV was inserted on the planning CT image by adding margins $(2-6 \mathrm{~mm})$ to the GTV according to respiratory movements measured by fluoroscopy. PTV was determined by adding $2 \mathrm{~mm}$ to the ITV. Normal liver was defined as the liver minus GTV. The four patients were informed regarding use of their clinical data for this study and provided written informed consent.

\section{Treatment plans}

At each institution, planning $\mathrm{CT}$ images and structure sets were imported into a radiotherapy treatment planning system (TPS), and study plans were created. The beam x-ray energy was set at $6 \mathrm{MV}$. Different dose calculation algorithms were allowed.

Routine clinical plans (practice plans) were established according to current treatment protocols at each institution, including prescription dose, prescription point and dose constraints. Another plan included prescribing 40 Gy in five fractions at $95 \%$ of the PTV (protocol plan 1). After analyzing and discussing the results of protocol plan 1, each institution was asked to implement an

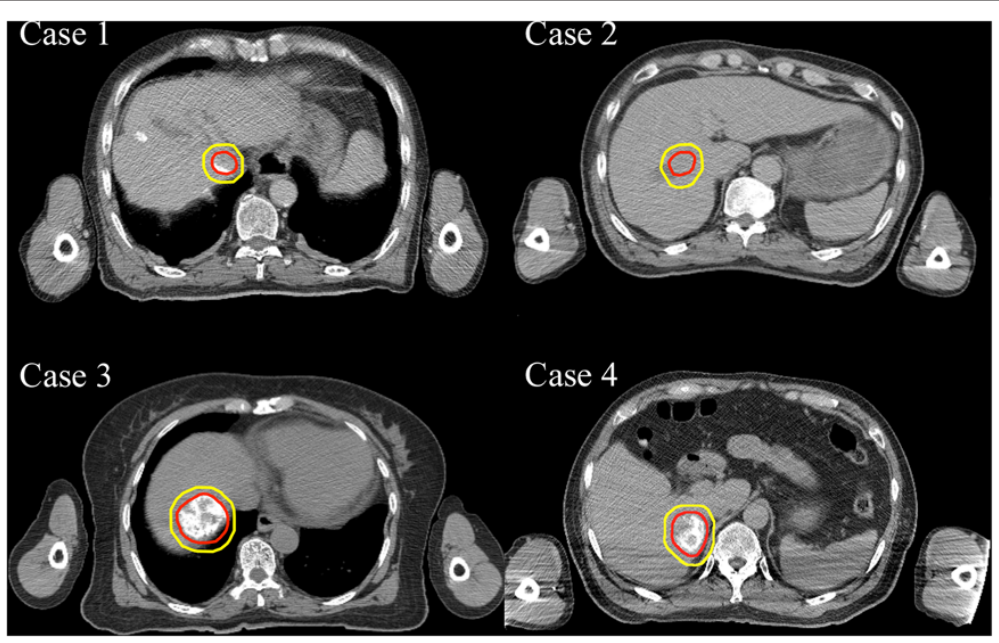

Figure 1 Planning computed tomography images. Outer and inner lines indicate the planning target volume and gross tumor volume, respectively. Case 1, hepatocellular carcinoma (23 mm) located in segment 1 (S1) near the duodenum; case 2, hepatocellular carcinoma (25 mm) located just below the diaphragm in S4; case 3, hepatocellular carcinoma (22 $\mathrm{mm}$ ) located in $\mathrm{S} 5$ near the inferior vena cava and the duodenum; and case 4, hepatocellular carcinoma (40 mm) located in S6/7. 
additional plan in which 40 Gy in five fractions was prescribed at the $70 \%$ isodose line of the global maximum dose within the PTV in which 95\% of the PTV received more than 40 Gy (protocol plan 2).

\section{Plan comparisons}

Planning CT images, structure sets, plans and doses from each institution were collected and imported to the treatment planning system (Eclipse, version 10.0, Varian, Palo Alto, CA). The following items were also collected and compared: radiotherapy unit, radiotherapy TPS, dose calculation algorithm, prescription dose, prescription point, beam arrangement, planning CT methods, target volume delineation methods and dose constraints. Dose volume histograms (DVHs) of the GTV, PTV and normal liver from each plan at each institution were evaluated. Median D50 ( $\left.\mathrm{D}_{\mathrm{m}} 50\right), \mathrm{D}_{\mathrm{m}} 90, \mathrm{D}_{\mathrm{m}} 98$, maximum dose and minimum dose were acquired. Median normal liver volume receiving 20 Gy or higher $\left(\mathrm{V}_{\mathrm{m}} 20\right)$ and median mean normal liver dose $\left(M_{L} D_{m}\right)$ were used to evaluate the normal liver dose. For GTV and PTV, the homogeneity index (HI) was defined as the maximum dose delivered to $2 \%$ of the target volume (D2) minus D98 divided by D50. Dose conformity was evaluated in terms of conformation number $(\mathrm{CN})$ [10], quantified as:

$$
\left(\mathrm{V}_{\mathrm{T}, \mathrm{pi}} / \mathrm{V}_{\mathrm{T}}\right) *\left(\mathrm{~V}_{\mathrm{T}, \mathrm{pi}} / \mathrm{V}_{\mathrm{pi}}\right)
$$

where: $\mathrm{V}_{\mathrm{T}, \mathrm{pi}}=$ volume within the PTV receiving a dose $\geq$ the prescription dose, $\mathrm{V}_{\mathrm{pi}}=$ volume receiving a dose $\geq$ the prescription dose, $\mathrm{V}_{\mathrm{T}}=\mathrm{PTV}$. We had defined metrics for planning evaluation before protocol plan 1, therefore we used same metrics to evaluate protocol plan 1 and 2.

\section{Results}

Current planning and treatment protocols at participating institutions are shown in Table 1. Remarkable variations between each institution were observed. Institutions A and B used non-coplanar and coplanar dynamic conformal arc beams, respectively. In contrast, institutions $\mathrm{C}$ and D used non-coplanar static beams. At institution A, the prescribed dose was to the $70 \%$ isodose line within the

Table 1 Planning and treatment parameters

\begin{tabular}{|c|c|c|c|c|}
\hline & A & B & C & D \\
\hline TPS & $\mathrm{XiO}$ & iPlan & iPlan & Pinnacle \\
\hline Calculation algorithm & SuperPosition & Pencil Beam & $\mathrm{XVMC}$ & CCCS \\
\hline \multirow[t]{2}{*}{ Prescription dose } & ChildA: 40Gy/5fr & $44 \mathrm{~Gy} / 4 \mathrm{fr}$ & Peripheral: 48Gy/4fr & Peripheral: 48Gy/4fr \\
\hline & ChildB: 35Gy/5fr & & PeriGl:60Gy/8fr & Cenrtral:60Gy/8fr \\
\hline Prescription point & $70 \%$ isodose & Isocenter & Isocenter & Isocenter \\
\hline \multirow[t]{2}{*}{ Beam arrangement } & Dynamic conformal arc & Dynamic conformal arc & Static & Static \\
\hline & Non-coplanar, 8 arcs & Coplanar, 2 arcs & Non-coplanar, 6-8 beams & Non-coplanar, 6-8 arcs \\
\hline \multirow[t]{2}{*}{$\begin{array}{l}\text { Respiratory } \\
\text { movement }\end{array}$} & $\begin{array}{l}\text { Confined free-breathing } \\
(<1 \mathrm{~cm})\end{array}$ & Confined free-breathing & Confined free-breathing & Gated by $\mathrm{Abches}^{\oplus}$ or RPM \\
\hline & $\begin{array}{l}\text { Under abdominal } \\
\text { compression }\end{array}$ & $\begin{array}{l}\text { Under abdominal } \\
\text { compression }\end{array}$ & Under abdominal compression & \\
\hline Target definition $\mathrm{CT}$ & Fusion of LSTCT + dynamic CT & Fusion of 4 times breathing $C T$ & Fusion of 4DCT + breath-hold CT & Breath-hold CT \\
\hline Planning CT & LSTCT & LSTCT & AvelP from $4 \mathrm{DCT}$ & Breath-hold CT \\
\hline ITV & $\mathrm{GTV}+2-6 \mathrm{~mm}$ & Fusion of 4 times breathing $\mathrm{CT}$ & Directly visualized by LSTCT & $\mathrm{ITV}=\mathrm{GTV}$ \\
\hline PTV & $\mathrm{ITV}+2 \mathrm{~mm}$ & ITV + CC10mm, AP, LR8mm & $\mathrm{ITV}+5 \mathrm{~mm}$ & ITV + CC8mm, AP, LR5 mm \\
\hline \multicolumn{5}{|l|}{ Dose constraints } \\
\hline Liver & $V_{20}($ include PTV $)<20 \%$ & $V_{<16}>700 \mathrm{cc}$ & $V_{20}<25 \% V_{<15}>700 \mathrm{cc}$ & $V_{20} \leqq 25 \%$ \\
\hline Spinal cord & $D_{\max }<25 G y, D_{1 c c}<20 G y$ & $\mathrm{D}_{\max }<25 \mathrm{~Gy} / 4 \mathrm{fr}$ & $\mathrm{D}_{\max }<25 \mathrm{~Gy} / 4 \mathrm{fr}$ & $\mathrm{D}_{\max }<25 \mathrm{~Gy} / 4 \mathrm{fr}$ \\
\hline Gl tract & $D_{\max }<25 G y, D_{1 c c}<20 G y$ & $\mathrm{D}_{10 \mathrm{cc}}<35 \mathrm{~Gy} / 4 \mathrm{fr}$ & $\mathrm{D}_{1 \mathrm{cc}}<40 \mathrm{~Gy} / 4 \mathrm{fr}, \mathrm{D}_{10 \mathrm{ccc}}<35 \mathrm{~Gy} / 4 \mathrm{fr}$ & $\begin{array}{l}\mathrm{D}_{1 c c}<30 \mathrm{~Gy} / 4 \mathrm{fr}, \mathrm{D}_{10 \mathrm{cc}} \\
<24 \mathrm{~Gy} / 4 \mathrm{fr}\end{array}$ \\
\hline Kidney & - & $V_{20}<30 \%$ & $V_{20}<30 \%$ & $\mathrm{D}_{1 / 3}<20 \mathrm{~Gy} / 4 \mathrm{fr}$ \\
\hline Heart & - & - & - & $\mathrm{D}_{1 / 3}<28 \mathrm{~Gy} / 4 \mathrm{fr}$ \\
\hline Gallbladder & - & - & - & $\mathrm{D}_{1 / 3}<40 \mathrm{~Gy} / 4 \mathrm{fr}$ \\
\hline
\end{tabular}

Abbreviations: TPS, treatment planning system; XVMC, X-ray voxel Monte Carlo algorithm; CCCS, collapsed cone convolution superposition; periGI perigastrointestinal; RPM, The Varian ${ }^{\circledast}$ Real-time Position Management ${ }^{\text {TM }}$ system; Gl, gastrointestinal; LSTCT, long scan time computed tomography; $4 D C T$, 4-dimensional computed tomography; AveIP, average intensity projection; GTV, gross tumor volume; PTV, planning target volume; ITV, internal target volume; $C C$, cranial and caudal; $L R A$, left, right and anterior; $P$, posterior; $L R$, left and right; $A P$, anterior and posterior. 
PTV surface, while the other three institutions prescribed to an isocenter.

\section{Practice plan}

GTV, PTV and normal liver DVHs for case 4 are shown in Figure 2a-c. The shoulder of the PTV DVH had a lower gradient and higher maximum dose at institution A compared to the other institutions. There was significant variation among DVHs in target volume, although low variation was observed in the normal liver. $D_{m} 95$ and $\mathrm{D}_{\mathrm{m}} 50$ of PTV of the four cases were $43.5 \mathrm{~Gy}$ (range, 41.1-46.5 Gy) and 48.4 Gy (range, 43.6-51.2 Gy), respectively. $\mathrm{V}_{\mathrm{m}} 20$ and $\mathrm{MLD}_{\mathrm{m}}$ were $15.9 \%$ (range, $12.2-18.9 \%$ ) and $10.8 \%$ (range, $8.8-12.5 \%$ ), respectively. Median HI was higher at institution A (Table 2, Figure 3).

\section{Protocol plan 1}

In protocol plan 1 (Table 2, Figure 2d-f), all of the institutions used a D95 of 40 Gy in all cases. Except for GTV and PTV DVHs at institution A, there was little variation in GTV, PTV and normal liver DVHs at each institution. PTV $\mathrm{D}_{\mathrm{m}} 50$ at institution A was higher than at the other institutions. $\mathrm{V}_{\mathrm{m}} 20$ and $\mathrm{MLD}_{\mathrm{m}}$ were $13.9 \%$ (range, 11.717.5\%) and 9.8 Gy (range, 8.8-11.4 Gy), respectively. HI was higher at institution A (Table 2, Figure 3). $\mathrm{CN}$ at institution $\mathrm{C}$ was relatively lower than at the other three institutions.

\section{Protocol plan 2}

In protocol plan 2 (Table 2, Figure 2g-i), all of the institutions complied with the dose constraints. Regarding variation among GTV and PTV DVHs in protocol plan 2 , the range of DVH values was reduced compared with protocol plan 1. Although the DVH shape was similar to the shape observed in the practice plan and protocol plan $1, V_{m} 20$ and $M L D_{m}$ were lower than the other two plans. Dose distribution at each institution is shown in Figure 4. Median $\mathrm{HI}$ and $\mathrm{CN}$ values were nearly equivalent at all institutions (Table 2, Figure 3).

\section{Discussion}

SABR is expected to be a treatment option indicated for HCC patients who are ineligible for surgery or radiofrequency ablation. However, various dose prescription and treatment planning strategies are currently used by different groups [3,4,7-9] and an optimal dose has not been determined.

For trials involving advanced radiation therapy techniques, the minimum acceptable degree of protocol compliance must be described to mitigate unacceptable variation

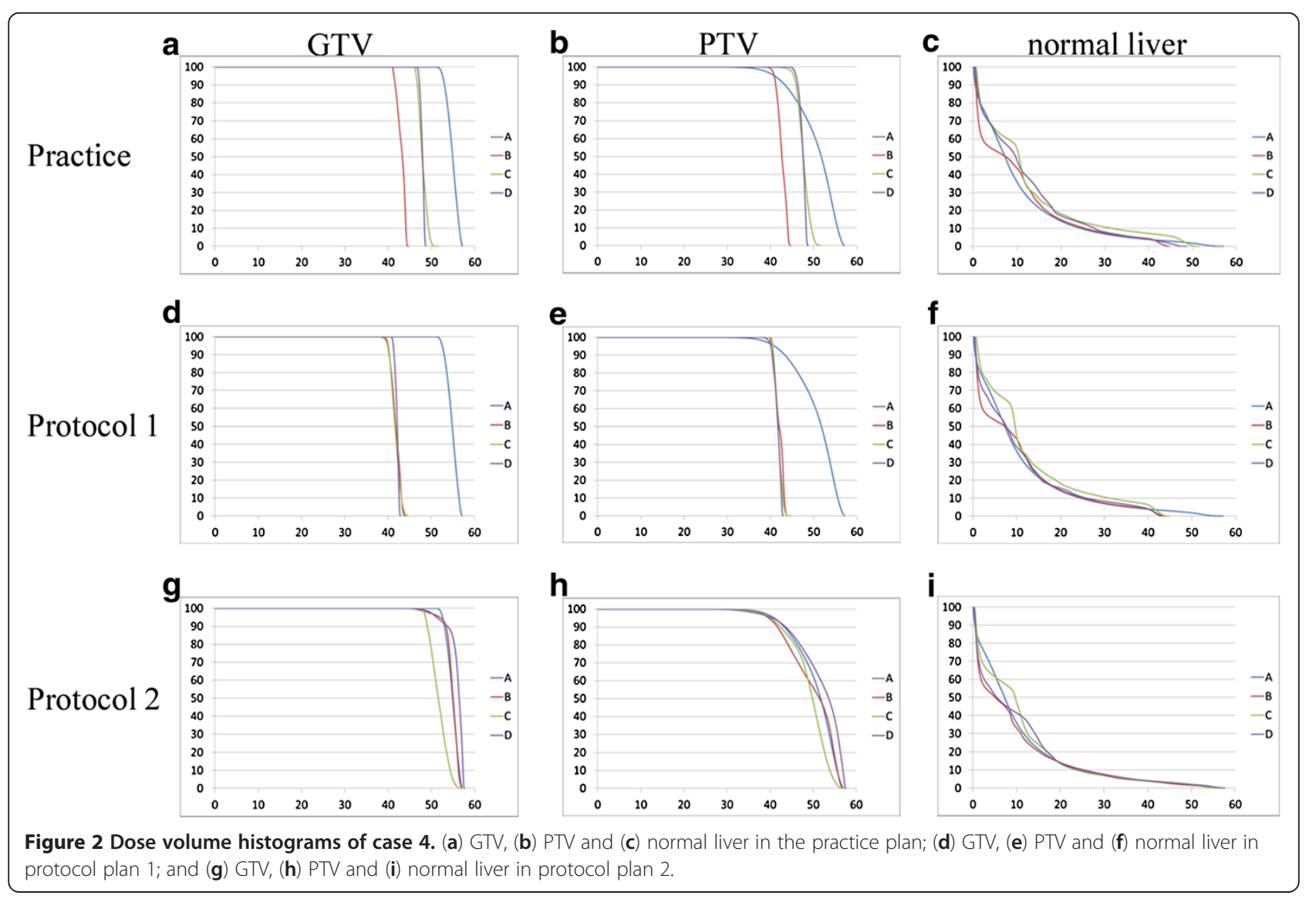


Table 2 Dose-volumetric data of target volumes

\begin{tabular}{|c|c|c|c|c|c|c|}
\hline \multirow{3}{*}{ Practice-plan } & & & \multirow[t]{2}{*}{ A } & \multirow{2}{*}{\multicolumn{2}{|c|}{$\begin{array}{l}\text { B } \\
\text { Median (Min - Max) }\end{array}$}} & \multirow[t]{2}{*}{ D } \\
\hline & & & & & & \\
\hline & & & & & & \\
\hline \multirow[t]{5}{*}{ GTV } & $\mathrm{D}_{\mathrm{m}} 50$ & (Gy) & $55.2(52.6-55.8)$ & $44.1(43.3-44.2)$ & $49.0(44.2-55.5)$ & $47.9(46.6-48.5)$ \\
\hline & $D_{m} 95$ & (Gy) & $53.1(48.8-54.2)$ & $42.7(41.3-43.7)$ & $47.6(44.2-54.3)$ & $47.2(46.6-47.8)$ \\
\hline & $D_{m} 98$ & (Gy) & $52.3(47.8-53.8)$ & $42.6(41.1-43.6)$ & $47.5(43.9-54.0)$ & $47.2(46.4-47.7)$ \\
\hline & Min & (Gy) & 50.0(49.3-53.1) & $42.2(40.9-43.3)$ & $46.8(43.1-53.4)$ & $46.3(39.6-47.0)$ \\
\hline & Max & (Gy) & $57.1(57.1-57.1)$ & $45.1(44.6-47.8)$ & $51.8(50.9-57.2)$ & $49.0(41.2-49.7)$ \\
\hline \multirow[t]{5}{*}{ PTV } & $\mathrm{D}_{\mathrm{m}} 50$ & (Gy) & $51.2(49.5-52.1)$ & $43.6(42.7-44.0)$ & 49.0(46.4-55.4) & $47.7(47.5-47.8)$ \\
\hline & $D_{m} 95$ & (Gy) & $41.2(41.0-41.5)$ & $41.1(40.7-41.9)$ & $46.5(43.2-53.4)$ & $45.7(45.5-46.5)$ \\
\hline & $D_{m} 98$ & (Gy) & 39.0(38.4-39.8) & $40.5(39.8-41.3)$ & $45.9(42.5-52.9)$ & $45.3(44.7-46.1)$ \\
\hline & Min & (Gy) & $32.5(27.5-34.0)$ & $37.1(35.4-37.3)$ & $40.5(37.7-50.7)$ & $43.7(41.9-45.0)$ \\
\hline & Max & (Gy) & $57.1(57.1-57.1)$ & $45.2(44.7-48.9)$ & $40.5(51.7-57.7)$ & $43.7(48.7-49.9)$ \\
\hline $\mathrm{HI}$ & & & $0.34(0.33-0.36)$ & $0.10(0.08-0.19)$ & $0.12(0.08-0.18)$ & $0.08(0.06-0.09)$ \\
\hline$V_{\mathrm{m}} 20$ (normal liver) & & (\%) & $13.2(6.7-14.1)$ & $12.2(6.5-14.9)$ & 18.9(13.7-22.3) & $18.5(10.1-23.2)$ \\
\hline $\mathrm{MLD}_{\mathrm{m}}$ & & (Gy) & $9.3(7.1-11.2)$ & $8.8(7.1-10.4)$ & $12.5(10.9-13.2)$ & $12.2(8.7-14.0)$ \\
\hline \multicolumn{7}{|l|}{ Protocol-plan1 } \\
\hline \multirow[t]{5}{*}{ GTV } & $D_{m} 50$ & (Gy) & $55.2(52.6-55.8)$ & $42.7(42.0-43.1)$ & $42.0(41.6-43.1)$ & $42.4(42.2-42.7)$ \\
\hline & $D_{m} 95$ & (Gy) & $53.1(48.8-54.2)$ & $41.6(40.2-42.0)$ & $40.8(40.6-41.2)$ & $44.5(41.2-42.7)$ \\
\hline & $\mathrm{D}_{\mathrm{m}} 98$ & (Gy) & $52.3(47.8-53.8)$ & $41.5(40.0-42.0)$ & $40.6(40.4-41.0)$ & $41.4(41.1-42.1)$ \\
\hline & Min & (Gy) & $50.0(49.0-53.0)$ & $41.0(39.7-42.0)$ & $39.9(39.6-40.4)$ & $41.0(40.7-42.0)$ \\
\hline & Max & (Gy) & $57.1(57.1-57.1)$ & $57.1(43.1-46.7)$ & $50.6(43.1-47.6)$ & $45.4(43.1-44.5)$ \\
\hline \multirow[t]{5}{*}{ PTV } & $\mathrm{D}_{\mathrm{m}} 50$ & (Gy) & $51.2(49.5-52.1)$ & $42.2(41.8-43.1)$ & $42.0(41.6-43.1)$ & $42.0(41.6-42.2)$ \\
\hline & $D_{m} 95$ & (Gy) & $41.2(41.0-41.5)$ & $40.0(40.0-40.1)$ & $40.1(40.1-40.2)$ & $40.0(40.0-40.2)$ \\
\hline & $D_{m} 98$ & (Gy) & $39.0(38.4-39.8)$ & 39.5(39.4-39.6) & 39.7(39.7-39.8) & $39.6(39.2-39.8)$ \\
\hline & Min & (Gy) & $32.5(27.5-34.0)$ & $36.4(34.1-37.5)$ & 38.9(38.6-39.1) & $37.3(36.6-40.7)$ \\
\hline & Max & (Gy) & $57.1(57.1-57.1)$ & $44.2(43.3-48.2)$ & $45.1(43.3-48.8)$ & $43.5(43.4-44.5)$ \\
\hline $\mathrm{HI}$ & & & $0.34(0.33-0.36)$ & $0.10(0.09-0.19)$ & $0.11(0.07-0.17)$ & $0.09(0.07-0.10)$ \\
\hline $\mathrm{CN}$ & & & $0.85(0.81-0.91)$ & $0.89(0.80-0.90)$ & $0.63(0.59-0.68)$ & $0.85(0.75-0.86)$ \\
\hline$V_{m} 20$ (normal liver) & & (\%) & 13.2(6.7-14.1) & 11.7(6.2-14.6) & 17.5(10.0-19.1) & $14.6(8.8-15.5)$ \\
\hline $\mathrm{MLD}_{\mathrm{m}}$ & & (Gy) & $9.3(7.1-11.2)$ & 8.8(6.9-9.9) & $11.4(8.6-12.3)$ & $10.2(7.6-10.4)$ \\
\hline \multicolumn{7}{|l|}{ Protocol-plan2 } \\
\hline \multirow[t]{5}{*}{ GTV } & $D_{m} 50$ & (Gy) & $55.2(52.6-55.8)$ & $55.3(53.2-56.3)$ & $53.6(51.6-55.0)$ & $56.2(55.9-56.6)$ \\
\hline & $\mathrm{D}_{\mathrm{m}} 95$ & (Gy) & $53.1(48.8-54.2)$ & $53.2(50.7-55.6)$ & $50.6(48.6-53.3)$ & $52.6(51.2-54.7)$ \\
\hline & $D_{m} 98$ & (Gy) & $52.3(47.8-53.8)$ & $52.2(47.4-55.3)$ & $50.1(48.0-52.8)$ & $50.3(47.4-53.0)$ \\
\hline & Min & (Gy) & $50.0(49.3-53.1)$ & $51.5(45.0-54.7)$ & $49.7(47.1-53.8)$ & $47.0(42.6-50.5)$ \\
\hline & Max & (Gy) & $57.1(57.1-57.1)$ & $57.1(57.1-57.1)$ & $57.1(57.1-57.1)$ & $57.1(57.1-57.1)$ \\
\hline \multirow[t]{5}{*}{ PTV } & $\mathrm{D}_{\mathrm{m}} 50$ & (Gy) & $51.2(49.5-52.1)$ & $52.5(51.5-54.0)$ & 51.0(49.9-52.3) & $53.1(50.8-53.8)$ \\
\hline & $D_{m} 95$ & (Gy) & $41.2(41.0-41.5)$ & $40.0(40.0-40.2)$ & $40.1(40.0-40.3)$ & $40.6(40.4-41.3)$ \\
\hline & $D_{m} 98$ & (Gy) & $39.0(38.4-39.8)$ & $37.1(36.7-37.7)$ & $37.8(37.4-38.2)$ & $38.5(36.3-38.9)$ \\
\hline & Min & (Gy) & $32.5(27.5-34.0)$ & 21.5(18.5-23.6) & $26.9(24.9-28.8)$ & $29.5(25.8-32.4)$ \\
\hline & Max & (Gy) & $57.1(57.1-57.1)$ & $57.1(57.1-57.1)$ & $57.1(57.1-57.1)$ & $57.1(57.1-57.1)$ \\
\hline
\end{tabular}


Table 2 Dose-volumetric data of target volumes (Continued)

\begin{tabular}{|c|c|c|c|c|c|}
\hline $\mathrm{HI}$ & & $0.34(0.33-0.36)$ & $0.37(0.36-0.38)$ & $0.36(0.35-0.39)$ & $0.35(0.35-0.39)$ \\
\hline $\mathrm{CN}$ & & $0.85(0.81-0.91)$ & $0.90(0.88-0.91)$ & $0.89(0.87-0.91)$ & $0.89(0.85-0.89)$ \\
\hline$V_{\mathrm{m}} 20$ (normal liver) & (\%) & 13.2(6.7-14.1) & $11.5(6.3-14.0)$ & $10.4(6.3-13.7)$ & $13.0(6.8-13.7)$ \\
\hline $\mathrm{MLD}_{\mathrm{m}}$ & (Gy) & $9.3(7.1-11.2)$ & 8.3(7.0-9.9) & $8.9(8.0-10.9)$ & $9.5(6.4-10.2)$ \\
\hline
\end{tabular}

Abbreviations: GTV, gross tumor volume; PTV, planning target volume; $H I$, homogeneity index; $C N$, conformation number; $M L D$, mean liver dose.

between institutions [11]. This study revealed many differences in planning and treatment protocols at several institutions (Table 1). In conducting a clinical trial of SABR, treatment planning can vary based on multiple factors, such as planning CT, target volume delineation, beam arrangement, dose calculation algorithms and prescription point [12]. It is difficult to unify the method to acquire planning $\mathrm{CT}$ because treatment modalities vary among institutions. In regard to measures to account for respiratory movement, it is important to set up some criteria with acceptable range in preparing for a protocol. Calculation algorithms have influence on dose distribution when some beams pass through materials with air density,

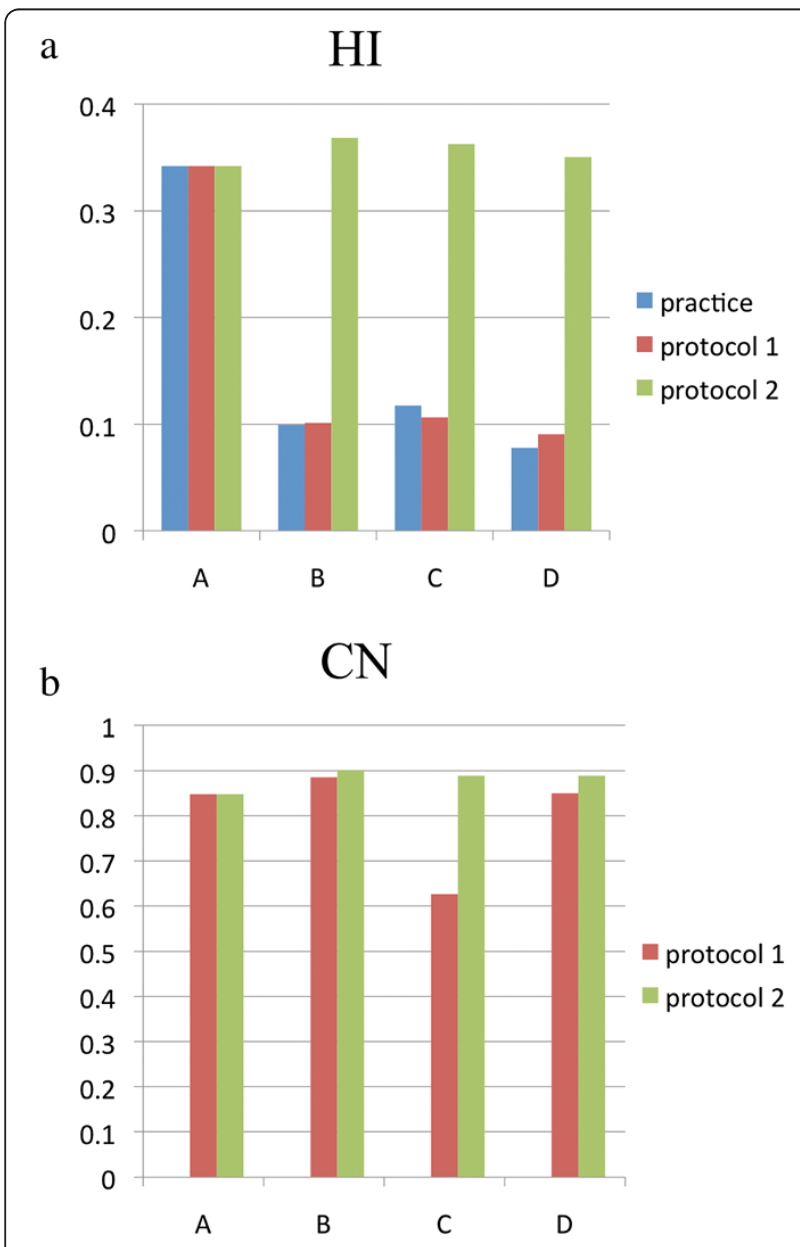

Figure 3 Homogeneity index $(\mathrm{HI})$ and conformation number (CN) at institutions A-D. Median (a) $\mathrm{HI}$ and (b) $\mathrm{CN}$ at institutions A-D. therefore newer generation calculation algorithms such as superposition or comparable algorithm may be preferable. Variations in target delineation have been reported by several investigators $[13,14]$. Delineation of HCC can also be affected by scanning protocol of triphasic CT, with or without use of MRI. In this study, identical target volumes were intentionally delineated prior to data distribution to eliminate variation and enable direct comparison of $\mathrm{DVH}$ parameters used in different planning methods.

In the practice plan, PTV dose distribution varied among institutions due to differences in prescription dose and prescription point. A uniform prescription dose of 40 Gy in five fractions administered as D95 were required in protocol plan 1. As a result, there was a significant gap between institution $\mathrm{A}$ and the other three institutions (Figure 2d-f) due to different prescription methods because institution A prescribed at the 70\% isodose level relative to the global maximum dose, while the other three institutions prescribed at the isocenter.

There are two different concepts regarding dose within the target in SABR. One maintains dose homogeneity within the target, which is generally prescribed at the isocenter. The concept has been widely utilized in Japan. In the other concept, dose is prescribed at the PTV margin and does not maintain dose homogeneity [15]. In the latter concept, there is another variation in prescription method which provides more flexibility and is more treatment planning system and technique independent. In a randomized phase III trial of Radiosurgery Or Surgery for operable Early stage (stage 1A) non-small cell Lung cancer (ROSEL) study, the dose prescription was based on D95 of the PTV receiving at least the nominal fraction dose, and D99 of the PTV receiving a minimum of $90 \%$ of the fraction dose. The dose maximum within the PTV should preferably be between $110 \%$ and $140 \%$ of the prescribed dose. The location of the treatment plan normalization point can be left to the institutions preference [16].

In conventional radiotherapy, International Commission on Radiation Units and Measurements (ICRU) Report 50 [17] recommends a uniform dose to the target volume within $-5 \%$ to $+7 \%$ of the prescribed dose with a radiation dose at the reference point, which is generally the isocenter. In contrast, dose heterogeneity within the target is acceptable in SABR for targets that do not involve functional normal tissue, as outlined in best practice guidelines 


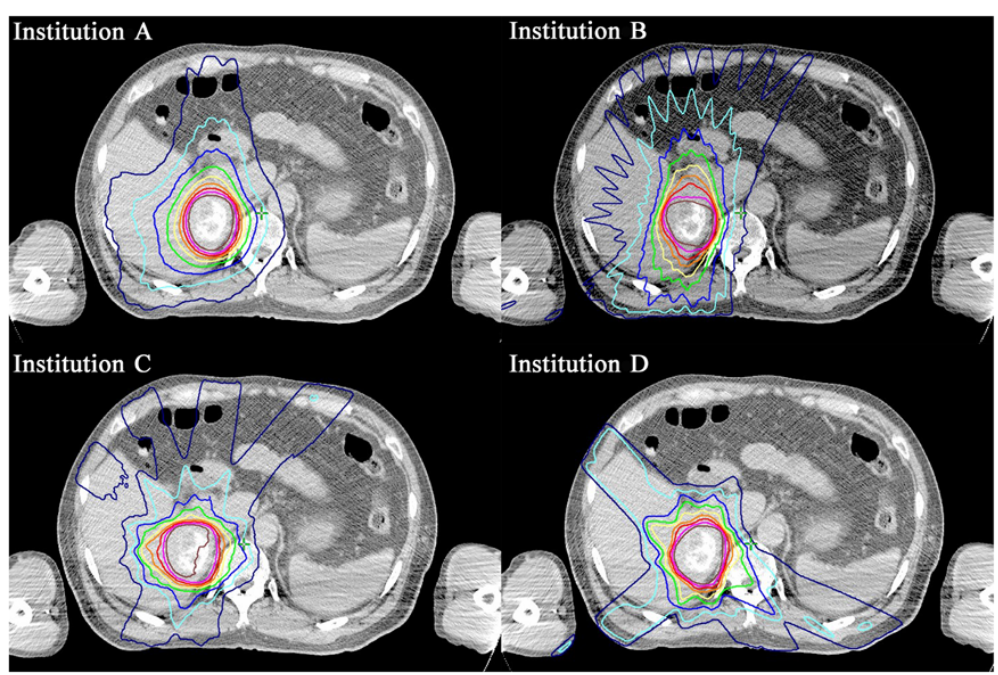

Figure 4 Dose distribution for case 4 in protocol plan 2.

by the American Association of Physicists in Medicine (AAPM) Task Group 101 [18]. By ignoring dose homogeneity within the PTV, tight conformity with steep and isotropic dose fall-off and high dose delivery to the target volume can be achieved in addition to a simultaneous reduction in the normal tissue dose [19]. In this study, institution A prescribed the dose at a $70 \%$ isodose line. Accordingly, protocol plan 2 required dosing to the $70 \%$ isodose line of the global maximum dose within $95 \%$ of the PTV. As a result, GTV and PTV doses were increased in protocol plan 2, while the normal liver dose decreased compared with protocol plan 1.

Improvements in DVH were primarily attributed to prescribing the dose at the $70 \%$ isodose line. Widder et al. [20] reported that dose prescription in SABR for lung cancer at isodose levels between $50 \%$ and $70 \%$ of the dose at the isocenter resulted in a lower dose to surrounding tissues and lungs compared with an $80 \%$ isodose level. Although there are no reports on optimal isodose levels for SABR to treat HCC, prescription to the $70 \%$ isodose level rather than an isocenter improved dose distribution in the current study.

Differences in DVH parameters between institutions, particularly in the V20 and MLD in the practice plan and protocol plan 1, were grouped according to static and dynamic beam arrangements. Institutions $\mathrm{A}$ and $\mathrm{B}$, which used a dynamic conformal arc, had lower V20 and MLD values than institutions $C$ and $D$, which used non-coplanar static beams. Although a greater number of beams generally results in better conformity and dose distribution gradients, six to eight non-coplanar static beams sufficiently fulfilled the planning requirement in protocol plan 2. Prescription at the $70 \%$ isodose line successfully reduced the dose to surrounding normal tissues regardless of different beam arrangements.
In addition to improving planning quality, the current study shared treatment strategies at various institutions. After data collection, researchers from the institutions discussed their treatment planning policies and compared study results. With respect to dose distribution at each institution (Figure 4), institution $C$ selected beam directions that increased non-irradiated normal liver volume as much as possible, while institutions A and B were not as concerned about low doses to the normal liver. Institution $\mathrm{D}$ indicated that avoiding as much of the gastrointestinal tract as possible rather than dose reduction in the normal liver was important. Multi-leaf collimator margin size also varied among institutions, from uniform margins around the PTV (generally 5 to $10 \mathrm{~mm}$ ) to variable margins in three-dimensional directions, due to different dose prescription policies. This information, which was discussed in person, can favorably influence researchers toward improved treatment planning. This study uncovered possible variations in SABR planning among participating institutions and would help to prepare for a comprehensive protocol as well as to define credentialing and evaluation criteria beforehand. In multi-center clinical trials, maintaining protocol treatment quality by minimizing these variations is a challenge. Therefore, this type of study prior to establishing a protocol is in agreement with the goals of quality assurance $(\mathrm{QA})$ programs that attempt to minimize variations. According to a meta-analysis and a systematic review, radiation therapy protocol deviations are associated with increased risk of treatment failure and overall mortality [21,22]. Well-organized QA programs will result in improved reliability of clinical trials and quality of practice [23].

As limitations, the current study only compared treatment planning methods directly related to SABR and did not consider other factors that could affect treatment, 
such as methods of planning CT acquisition, contouring of at-risk targets and organs, patient fixation and respiratory gating. Calculation algorithms were not a key focus, which could influence dose distribution under specific conditions. The impact of variations in calculation algorithms based on dose distribution should be further evaluated.

\section{Conclusion}

In planning SABR to treat $\mathrm{HCC}$, there were notable inter-institutional differences. When the dose was prescribed to an isodose line fitted to the PTV surface, prescription requirements were fulfilled and differences in DVH between institutions decreased significantly. In multi-institutional studies, detailed dose specifications based on collaboration are necessary. A thoroughly described protocol with a radiotherapy QA program will lead to high-quality treatment and reliable results.

\section{Competing interests}

AT received grant from Varian Research Collaboration Program. SI received fees from Elekta K.K. for consultancy for the company.

The other authors declare that they have no competing interests.

\section{Authors' contributions}

TE analyzed data, drafted and revised the manuscript. AT, NS, SI, TK, YM, YM, YI revised the manuscript critically. YO prepared patient data sets for this planning study, collected and analyzed data. AT, YO, TK, SO, TN, YM, MN, YM, SY performed treatment planning. All the authors participated in this study design and discussion all along this study, read and approved the final manuscript.

\section{Acknowledgement}

This work was supported in part by the National Cancer Center Research and Development Fund (23-A-21). AT received grant from Grant-in-Aid for Scientific Research (C) from the Japan Society for the Promotion of Science. Yl received grant from National Cancer Center Research and Development Fund.

\section{Author details}

${ }^{1}$ Radiation Oncology Center, Ofuna Chuo Hospital, Kanagawa, Japan. ${ }^{2}$ Department of Radiation Oncology, Juntendo University, Tokyo, Japan. ${ }^{3}$ Department of Radiology, Hiroshima University Graduate School of Biomedical Sciences, Hiroshima, Japan. ${ }^{4}$ Department of Radiation Oncology and Image-applied Therapy, Kyoto University, Kyoto, Japan. ${ }^{5}$ Department of Radiation Oncology, Niigata Cancer Center Hospital, Niigata, Japan. ${ }^{6}$ Department of Radiation Oncology, National Cancer Center Hospital, Tokyo, Japan.

Received: 8 March 2013 Accepted: 30 April 2013

Published: 4 May 2013

\section{References}

1. Bruix J, Sherman M: Management of hepatocellular carcinoma: an update. Hepatology (Baltimore, Md) 2011, 53:1020-1022.

2. European Association for the Study of the Liver, European Organisation for Research and Treatment of Cancer: EASL-EORTC clinical practice guidelines: management of hepatocellular carcinoma. J Hepatol 2012, 56:908-943.

3. Mendez Romero A, Wunderink W, Hussain SM, De Pooter JA, Heijmen BJ, Nowak PC, Nuyttens JJ, Brandwijk RP, Verhoef C, ljzermans JN, Levendag PC: Stereotactic body radiation therapy for primary and metastatic liver tumors: A single institution phase i-ii study. Acta oncologica (Stockholm, Sweden) 2006, 45:831-837.

4. Tse RV, Hawkins M, Lockwood G, Kim JJ, Cummings B, Knox J, Sherman M, Dawson LA: Phase I study of individualized stereotactic body radiotherapy for hepatocellular carcinoma and intrahepatic cholangiocarcinoma. J Clin Oncol J Am Soc Clin Oncol 2008, 26:657-664.

5. Lee MT, Kim JJ, Dinniwell R, Brierley J, Lockwood G, Wong R, Cummings B, Ringash J, Tse RV, Knox JJ, Dawson LA: Phase I study of individualized stereotactic body radiotherapy of liver metastases. J Clin Oncol J Am Soc Clin Oncol 2009, 27:1585-1591.

6. Cardenes HR, Price TR, Perkins SM, Maluccio M, Kwo P, Breen TE, Henderson MA, Schefter TE, Tudor K, Deluca J, Johnstone PA: Phase I feasibility trial of stereotactic body radiation therapy for primary hepatocellular carcinoma. Clin Trans Oncol Pub Feder Spanish Oncol Soc Nat Cancer Ins Mexico 2010, 12:218-225.

7. Louis C, Dewas S, Mirabel X, Lacornerie T, Adenis A, Bonodeau F, Lartigau E: Stereotactic radiotherapy of hepatocellular carcinoma: preliminary results. Technol Cancer Res Treat 2010, 9:479-487.

8. Kwon JH, Bae SH, Kim JY, Choi BO, Jang HS, Jang JW, Choi JY, Yoon SK, Chung KW: Long-term effect of stereotactic body radiation therapy for primary hepatocellular carcinoma ineligible for local ablation therapy or surgical resection. Stereotactic radiotherapy for liver cancer. BMC Cancer 2010, 10:475.

9. Andolino DL, Johnson CS, Maluccio M, Kwo P, Tector AJ, Zook J, Johnstone PA, Cardenes HR: Stereotactic body radiotherapy for primary hepatocellular carcinoma. Int J Radiation Oncol, Biol, Physics 2011, 81:e447-e453.

10. van't Riet $A$, Mak AC, Moerland MA, Elders $L H$, van der Zee W: A conformation number to quantify the degree of conformality in brachytherapy and external beam irradiation: application to the prostate. Int J Rad Oncol, Biol, Physics 1997, 37:731-736.

11. Fairchild A, Bar-Deroma R, Collette L, Haustermans K, Hurkmans C, Lacombe D, Maingon P, Poortmans P, Tomsej M, Weber DC, Gregoire V: Development of clinical trial protocols involving advanced radiation therapy techniques: the European Organisation for Research and Treatment of Cancer Radiation Oncology Group approach. European J Cancer (Oxford, England: 1990) 2012, 48:1048-1054.

12. Matsuo Y, Takayama K, Nagata Y, Kunieda E, Tateoka K, Ishizuka N, Mizowaki T, Norihisa Y, Sakamoto M, Narita Y, et al: Interinstitutional variations in planning for stereotactic body radiation therapy for lung cancer. Int J Rad Oncol, Biol, Physics 2007, 68:416-425.

13. Bowden P, Fisher R, Mac Manus M, Wirth A, Duchesne G, Millward M, McKenzie A, Andrews J, Ball D: Measurement of lung tumor volumes using three-dimensional computer planning software. Int J Rad Oncol, Biol, Physics 2002, 53:566-573.

14. Senan S, Van Sornsen De Koste J, Samson M, Tankink H, Jansen P, Nowak PJ, Krol AD, Schmitz P, Lagerwaard FJ: Evaluation of a target contouring protocol for 3D conformal radiotherapy in non-small cell lung cancer. Radiotherapy Oncol J European Soc Therapeutic Radiol Oncol 1999, 53:247-255

15. Nagata Y, Matsuo Y, Takayama K, Norihisa Y, Mizowaki T, Mitsumori M, Shibuya K, Yano S, Narita Y, Hiraoka M: Current status of stereotactic body radiotherapy for lung cancer. Int J Clin Oncol/Japan Soc Clin Oncol 2007, 12:3-7.

16. Hurkmans CW, Cuijpers JP, Lagerwaard FJ, Widder J, van der Heide UA, Schuring D, Senan S: Recommendations for implementing stereotactic radiotherapy in peripheral stage IA non-small cell lung cancer: report from the Quality Assurance Working Party of the randomised phase III ROSEL study. Radiation Oncol (London, England) 2009, 4:1.

17. ICRU: Report 50: Prescribing, recording and reporting photon beam therapy, Journal of ICRU. United Kingdom: Oxford University Press; 1993.

18. Benedict SH, Yenice KM, Followill D, Galvin JM, Hinson W, Kavanagh B, Keall P, Lovelock M, Meeks S, Papiez L, et al: Stereotactic body radiation therapy: the report of AAPM Task Group 101. Med Phys 2010, 37:4078-4101.

19. Oku Y, Takeda A, Kunieda E, Sudo Y, Oooka Y, Aoki Y, Shimouchi Y, Nishina R, Nomura K, Sugiura M, Ohashi T: Analysis of suitable prescribed isodose line fitting to planning target volume in stereotactic body radiotherapy using dynamic conformal multiple arc therapy. Practical Radiation Oncol 2012, 2:46-53.

20. Widder J, Hollander M, Ubbels JF, Bolt RA, Langendijk JA: Optimizing dose prescription in stereotactic body radiotherapy for lung tumours using Monte Carlo dose calculation. Radiotherapy Oncol J European Soc Therapeutic Radiol Oncol 2010, 94:42-46.

21. Weber DC, Tomsej M, Melidis C, Hurkmans CW: QA makes a clinical trial stronger: evidence-based medicine in radiation therapy. Radiotherapy Oncol J European Soc Therapeutic Radiol Oncol 2012, 105:4-8. 
22. Ohri N, Shen X, Dicker AP, Doyle LA, Harrison AS, Showalter TN: Radiotherapy protocol deviations and clinical outcomes: a meta-analysis of Cooperative Group Clinical Trials. J Nat Cancer Ins 2013, 105:387-393.

23. Bekelman JE, Deye JA, Vikram B, Bentzen SM, Bruner D, Curran WJ Jr, Dignam J, Efstathiou JA, FitzGerald TJ, Hurkmans C, et al: Redesigning radiotherapy quality assurance: opportunities to develop an efficient evidence-based system to support clinical trials-report of the National Cancer Institute Work Group on Radiotherapy Quality Assurance. Int J Radiation Oncol, Biol, Physics 2012, 83:782-790.

doi:10.1186/1748-717X-8-113

Cite this article as: Eriguchi et al:: Multi-institutional comparison of treatment planning using stereotactic ablative body radiotherapy for hepatocellular carcinoma - benchmark for a prospective multi-institutional study. Radiation Oncology 2013 8:113.

\section{Submit your next manuscript to BioMed Central and take full advantage of:}

- Convenient online submission

- Thorough peer review

- No space constraints or color figure charges

- Immediate publication on acceptance

- Inclusion in PubMed, CAS, Scopus and Google Scholar

- Research which is freely available for redistribution 